\title{
Robust Trilateration Based Algorithm for Indoor Positioning Systems
}

\author{
Simeon Pande and Kwame S Ibwe* \\ College of Information and Communication Technologies, University of Dar es Salaam, \\ P. O. Box 33335 Dar es Salaam, Tanzania. \\ *Corresponding author, e-mail: kwame.ibwe@gmail.com \\ Received 30 Apr 2021, Revised 30 Jul 2021, Accepted 31 Jul 2021, Published Aug 2021 \\ DOI: https://dx.doi.org/10.4314/tjs.v47i3.28
}

\begin{abstract}
Indoor Positioning Systems (IPS) plays crucial roles in indoor environment items positioning used in self-navigating robots and helping hands. To obtain position information, positioning algorithms employing Received Signal Strength Indicator (RSSI) are of great benefits since they reuse the existing radio wireless infrastructures for indoor positioning. However, the changes in the indoor environment decrease the overall accuracy of the developed indoor positioning algorithms. To cope with the challenge of environmental dependency in indoor positioning, a robust algorithm using radio signal identification was developed. The algorithm uses circle expansion and reduction mechanism to achieve better RSSI-Distance relationship. The distances from RSSI-Distance relationship are used in trilateration algorithm for position estimation. Experiments were performed to compare position accuracy of the basic RSSI-Based and the proposed algorithm. Simulation results showed that proposed algorithm showed less average positioning errors by $11.2066 \%$ and $3.7279 \%$ at path loss coefficients of 3.11 and 3.21 , respectively compared to the existing algorithms. Likewise, the proposed algorithm showed $2.7282 \%$ increase in positioning error when environment was changed from that of path loss coefficient 3.11 to 3.21. The existing basic algorithms show error fluctuation of $10 \%$ with the same environment changes.
\end{abstract}

Keywords: Indoor Positioning System, RFID, RSSI, Trilateration.

\section{Introduction}

There is a long standing interest in ubiquitous positioning (the ability to determine a position in any environment) in both outdoors and indoors environment. Global Positioning System (GPS) is widely used in positioning and navigation systems due to its accuracy and ease of deployment. But the reliability and efficiency of GPS is limited to environments where line-of-sight signal paths can be achieved between transmitting satellite and target device (Tamas and Toth 2019). In environments where line-of-sight signal path cannot be established, GPS is prone to erroneous readings leading to inaccurate estimations. Hence, accurate positioning in such environments demands indoor positioning systems capable of combating the multipath effects to give accurate estimations (Maheepala et al. 2020). The desire has increased in recent years due to the introduction of Industry 4.0 (Carrasco et al. 2018). Industry 4.0 represents the phenomenon of Smart Factories which include artificial intelligence, robotics, big data, machine-to-machine interfaces and the Internet of Things (IoT) technologies. These technologies have sparked many researchers to advance into the development of indoor positioning systems to accommodate location aware services (Maung and Zaw 2020). These systems find applications from asset tracking, intelligent traffic control, indoor item 
positioning robots, self-navigating robots (cars) and coffee serving robots. Therefore, indoor positioning is fundamental for smart factories, smart warehouses and smart buildings. However, multipath propagation characteristics and interferences in the indoor environment cause high variability of Received Signal Strength (RSS), resulting in higher positioning errors (Maheepala et al. 2020).

To meet the requirements for inside buildings and minimize the positioning errors, different radio technologies have been proposed by researchers. Infrared (Blankenbach et al. 2012), ultrasonic (Yayan et al. 2015), Bluetooth (Bekkelien 2012), WiFi (Yang and Shao 2015), magnetic field (Kim et al. 2017) and Radio Frequency Identification (RFID) (Bai et al. 2012, Zhang et al. 2017) have been proposed and developed in order to provide positioning and navigation in indoor environment.

Ultrasonic indoor positioning systems offer a number of advantages over other systems in terms of low system costs, reliability, scalability, high energy efficiency, and most importantly, zero leakage between rooms. It provides fine-grained location with centimeter level accuracy. Ultrasonic systems can also track multiple mobile nodes at one time, that is, high capacity of location system to serve many users simultaneously (Yayan et al. 2015). However, these systems rely on time of arrival (TOA) measurement of the signal, calculated using velocity of sound. But unlike radio frequency (RF) signals, velocity of sound in air does not remain constant and varies largely with environmental conditions, especially humidity and temperature. High humidity causes the signal to fade away quickly and travel short distance making the systems highly vulnerable to estimation errors (Carotenuto et al. 2018).

Infrared (IR) indoor positioning systems require a direct line of sight between sender and receiver. The IR based indoor positioning systems have weak security and privacy protection settings. The IR signals are easily distorted with interference from fluorescent light and sunlight. Beside this, the IR based indoor system has expensive system hardware and maintenance costs (Blankenbach et al. 2012). Li (2014) provided theoretical details for 3D indoor positioning using least square method for Bluetooth signals. However, the results from simulation and hardware experiments showed that the average of absolute precision of location estimation is at meter level (Chai et al. 2016). Authors in Kim et al. (2017) proposed an indoor positioning system with a particle filter system where the weights of particles are updated by multiple magnetic sensors and three magnetic field maps: a horizontal intensity map, a vertical intensity map, and a direction information map. The system integrates magnetic field map navigation and an encoder system. The results showed that the accuracy of the estimated position of the proposed system is at meter level. In Chao et al. (2016), the authors proposed a visual control interface for a mobile robot with a single camera to estimate the $3 \mathrm{D}$ position of a target. They used $\mathrm{Wi}-\mathrm{Fi}$ to transmit the control signal and video from the mobile robot. However, the accuracy of the proposed 3D estimation method was in order of two meters (Dao et al. 2014, Chen et al. 2015).

Radio frequency identification (RFID) is the key technology necessary to realize the Internet of Things (IoT) and cyber-physical systems (CPS), widely used in health monitors, smart homes, smart cities, vehicle location, construction, supply chain management and object tracking (Deng et al. 2016, Xu et al. 2017). RFID positioning is based on Received Signal Strength Indicator (RSSI). RFID has the advantages of low cost, long life, low power consumption and easy deployment, which attracts many researchers to use it in indoor environments. Compared with ultrasonic, Wi$\mathrm{Fi}$ and Bluetooth, RFID used in indoor positioning is more energy efficient (Farahani 2008, Maung and Zaw 2020).

Larranaga et al. (2010) proposed a robust, flexible and easy to deploy indoor positioning algorithm based on RSSI values for positioning and calibration phase. To address the 
challenging nature of the environment, the algorithm used matrices calibrations when the blind node is located. The environmental parameters are updated on every calibration phase taking account of the last position estimation. The authors managed to obtain an average error of $2.34 \mathrm{~m}, 1.74 \mathrm{~m}$ and $4.24 \mathrm{~m}$ on a big, small and corridor rooms, respectively. The total average position error for all of the rooms was $2.77 \mathrm{~m}$. Despite the authors' good positioning algorithm, it is clear that the method had a tradeoff on position accuracy.

The authors in Zhang et al. (2012) presented a novel RSSI-based adaptive algorithm for wireless sensor networks (WSN). The algorithm works by separating the whole testing group into different pieces and assigning characteristic node in every region, one of the two models (empirical and theoretical models) is chosen after matching the error between the actual distance, the RSSI and the distance calculated from the two models by the characteristic node to minimize the positioning error. At any particular moment, the algorithm with less position error is used to present overall results. Since the indoor environments have infinity changes, this method will not give accurate results on unforeseen environment changes (Hausman and Januszkiewicz 2014, Ge et al. 2015, Kaluža et al. 2017).

The author in Xu et al. (2013) presented the WSN localization algorithm based RSSI differential correlation which positions a correction node close to the blind nodes and applies the correction factor and difference coefficient to correct the distance measurement results. The algorithm was implemented in 100 $\mathrm{m}$ by $100 \mathrm{~m}$ area and managed to achieve absolute error of $1.3 \mathrm{~m}$ with respect to chosen nine points in the area. The authors achieved accurate results on a large area but the overall accuracy of the algorithm heavily depends on the reading on the correction node. If the correction node fails to deliver accurate measurements due to change of environments on the area close to the correction node, the algorithm will accumulate more position errors
(Huynh et al. 2014, Huang et al. 2015, Helander and Humpus 2015).

The authors in Hussein et al. (2015) expressed the effects of physical barrier attack (presence of an obstacle to cause environment change) on mobile target through a distributed sensor network. The barrier confuses the localization process and adds localization error. The authors proposed an algorithm that uses two techniques of multiple frequencies and power levels identified with averaging the received power levels all over the transmitted frequencies in order to mitigate the shadowing effects in the wireless channel propagation. The author managed to obtain the minimum average position error of $3.2 \mathrm{~m}$ and $3.4 \mathrm{~m}$ for path loss coefficient equal to 3 and 4 using trilateration algorithm.

The authors in Liu et al. (2016) proposed RFID based indoor positioning algorithm using reference tags to estimate the location according to the measured RSSI compared with the RSSI value recorded in the database. However, the multipath effects in the various indoor environments significantly affect the positioning accuracy and the overall accuracy of the algorithm (Li 2014, Leitinger et al. 2014, Lee et al. 2016, Kim et al. 2017). The authors in Xu et al. (2017) extended the work in Liu et al. (2016) and achieved estimation accuracy by $10 \%$.

Tamas and Toth (2019) proposed a topologybased application-specific classification evaluation method for symbolic indoor positioning. It used gravitational force-based approach defined in such a way that the classification error be proportional to the sizes of the rooms. Due to environment-specific limitation of this method, it is challenging to generalize for indoor environments which constantly change (Lu et al. 2010, Liu et al. 2014, Liu et al. 2016). Authors in Sangthong et al. (2020) presented a new method to evaluate the WSN technology for the indoor localization using the weight range localizer (WRL) and relative span exponential weight range localizer (RS-WRL) based on RSSI to estimate 
the position of target node. However, the average position error is $1.9 \mathrm{~m}$.

Different researchers have proposed different solutions to achieve higher position accuracy in indoor positioning. However, environment dependency is still a challenge. This is because the indoor environments have been proved to be challenging to model due to the nature and activities that define them which are constantly changing. The changes in indoor environment are inevitable which decreases the overall accuracy of the developed indoor positioning algorithms. To address the challenge, a robust indoor positioning algorithm that is environment independent is developed in this work. The algorithm uses trilateration algorithm with circles expansion and reduction method to achieve the best position estimates. The contributions include the ability of the proposed algorithm to re-iterate to the optimal intersection of the reference RFID tags where the RSSI is theoretically high, hence counter the environmental and multipath degradation to signal power. The developed algorithm opens up for further cutting-edge applications in indoor environment by applying the proposed methods to remove environment dependency. A comparison of existing RSSI-based indoor positioning algorithms is done to obtain the estimation of distance error performance capabilities. The distance error performance is assessed in terms of positioning accuracy in meters. These results are obtained using $\mathrm{C}++$ simulation platform.

\section{Materials and Methods \\ Trilateration algorithm}

Lateration is a positioning algorithm which computes coordinate position from the distances between the reference positions and targeted positions ( $\mathrm{Pu}$ et al. 2011, Tsang et al. 2015). Trilateration is a special kind of lateration which uses three reference nodes to calculate the position on the unknown node. Trilateration algorithm uses RSSI measurements to estimate the distance between the (targeted node) tag and (reference node) reader. The distances between reference locations and the target location can be considered as the radii of many circles with centers at every reference location. Hence, the target location is the intersection of all the sphere surfaces as shown in Figure 1 (Alarifi et al. 2016).

In Figure 1, three reference nodes are randomly allocated. A target node is allowed to move around the reference nodes. The target node $\left(T_{1}\right)$ can be positioned using the coordinates of the reference nodes $\left(\mathrm{R}_{1}, \mathrm{R}_{2}\right.$, and $\left.\mathrm{R}_{3}\right)$ and the distances $\left(d_{1}, d_{2}, d_{3}\right)$ between the target nodes and the reference node $(\mathrm{Xu}$ et al. 2016). 


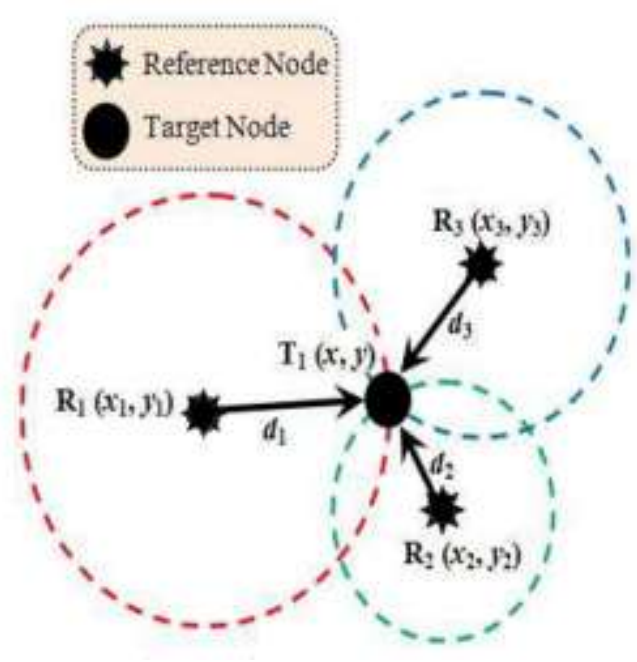

Figure 1: Trilateration estimation (Pu et al. 2011).

A simple solution can be achieved using Pythagoras theorem as

$$
\begin{aligned}
& d_{1}^{2}=\left(x_{1}-x\right)^{2}+\left(y_{1}-y\right)^{2} \\
& d_{2}^{2}=\left(x_{2}-x\right)^{2}+\left(y_{2}-y\right)^{2} \\
& d_{3}^{2}=\left(x_{3}-x\right)^{2}+\left(y_{3}-y\right)^{2}
\end{aligned}
$$

Rearranging Equation 1 and solving for $x$ and $y$, the position coordinate of the target node can be obtained as

$$
\begin{aligned}
& x=\frac{A Y_{32}+B Y_{13}+C Y_{21}}{2\left(x_{1} Y_{32}+x_{2} Y_{13}+x_{3} Y_{21}\right)} \\
& y=\frac{A X_{32}+B X_{13}+C X_{21}}{2\left(x_{1} X_{32}+x_{2} X_{13}+x_{3} X_{21}\right)}
\end{aligned}
$$

whereby

$$
\begin{aligned}
& A=x_{1}^{2}+y_{1}^{2}-d_{1}^{2} \\
& B=x_{2}^{2}+y_{2}^{2}-d_{2}^{2} \\
& C=x_{3}^{2}+y_{3}^{2}-d_{3}^{2}
\end{aligned}
$$

$$
\begin{aligned}
& X_{32}=x_{3}-x_{2} \\
& X_{13}=x_{1}-x_{3} \\
& X_{21}=x_{2}-x_{1} \\
& Y_{32}=y_{3}-y_{2} \\
& Y_{13}=y_{1}-y_{3} \\
& Y_{21}=y_{2}-y_{1}
\end{aligned}
$$

To use lateration techniques, at least three reference nodes are required. The assumption in traditional trilateration method is the intersection of all three or more circles (Pu et al. 2011, Huyn et al. 2014, Xu et al. 2016). The constantly changing indoor environment makes the edge to edge intersection of all reference circles challenging to achieve in practical conditions. The dependency of edge to edge intersection of all three or more circles deteriorates the estimation accuracy of trilateration techniques. This work develops the algorithm that is independent of the indoor environment and the edge to edge intersections of the reference nodes. 


\section{RSSI-Distance relationship}

In this work, the tags arrangement used in Javaid et al. (2015) was adopted. The indoor measurements area is $6 \mathrm{~m} \times 4 \mathrm{~m}$. Three reference tags are chosen from the total received number of reference tags in the environment as shown in Figure 2. Figure 2 describes the arrangement of the reference tags Anchor Node 1 (Tag 1), Anchor Node 2 (Tag 2) and Anchor Node 3 (Tag 3) positioned at points $(1,3),(3,5)$ and $(3,1)$, respectively, while the target Reader ( $\mathrm{T}$ ) can be positioned on different points on the $6 \times 4$ square meter indoor environment. The receiving reader receives the tag identification number (Tag ID) and measures its signal strength (RSSI). The reader then arranges RSSI values in descending order, starting with the largest RSSI value with their respective Tag IDs. The strongest signal gives better position estimation. The respective distances are calculated and sent to positioning algorithm.

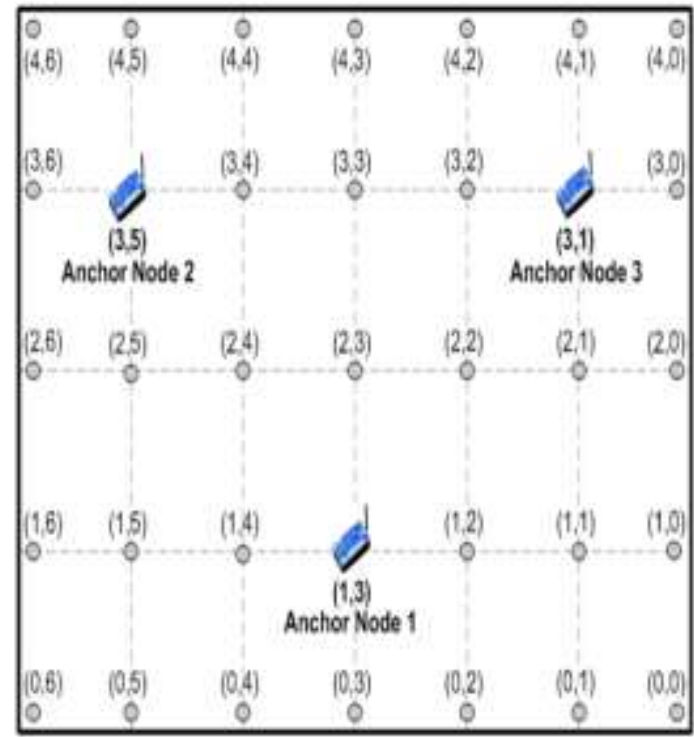

Figure 2: RFID Tags and readers arrangement in a $6 \mathrm{~m}$ x $4 \mathrm{~m}$ room (Javaid et al. 2015).

From the arrangement in Figure 2, the position of an arbitrary target tag in the two dimensional $(x, y)$ space, could be summarized as shown in Figure 3. The target tag location can be estimated using Equation 1 as

$$
\begin{aligned}
& d_{2}^{2}=(3-x)^{2}+(5-y)^{2} \\
& d_{3}^{2}=(3-x)^{2}+(1-y)^{2}
\end{aligned}
$$




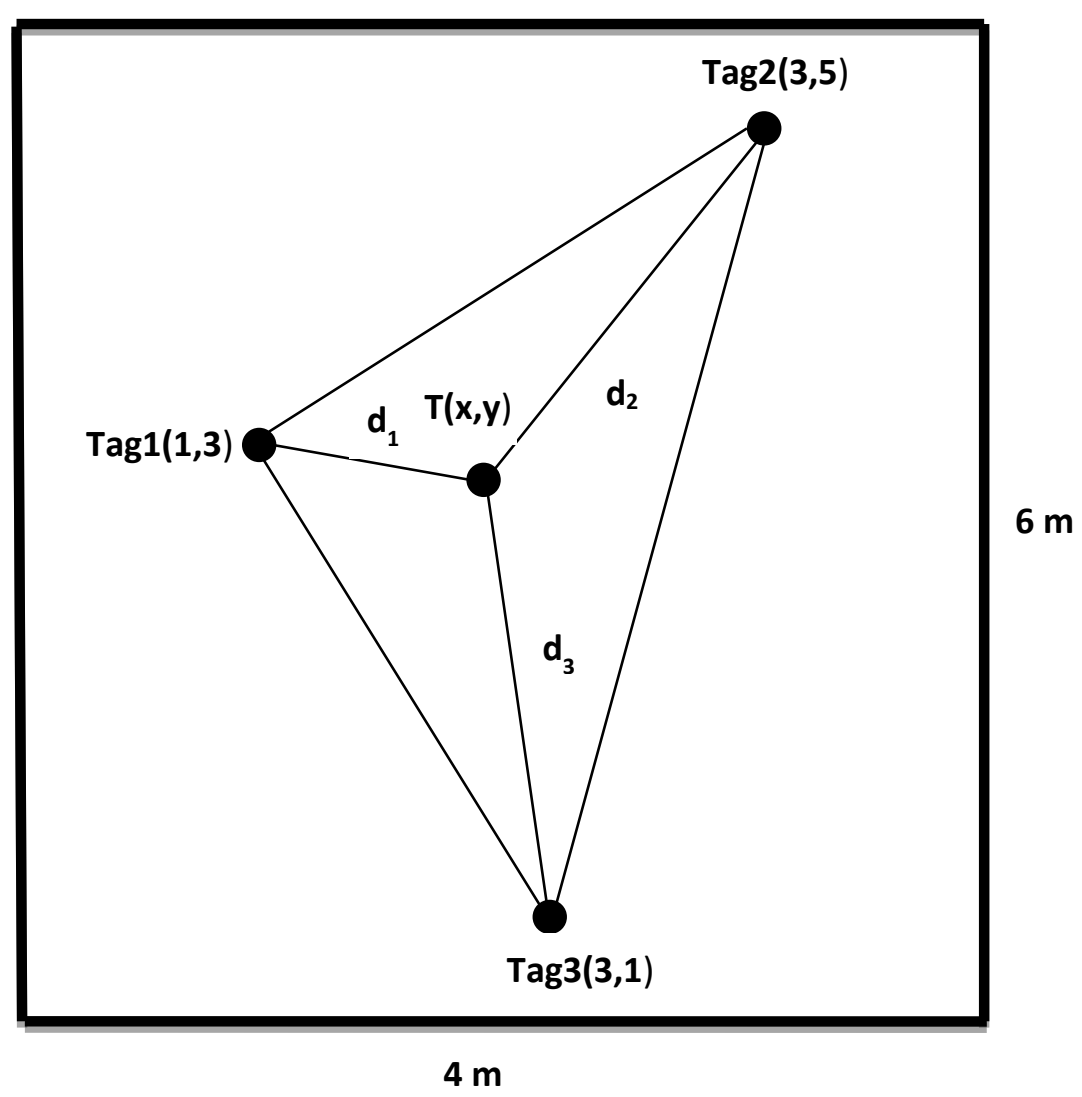

Figure 3: Summary arrangement of target and reference RFID tags.

Solving for $\mathrm{x}$ and $\mathrm{y}$ using Equations 5, 6 and 7, the values could be estimated as

$$
\begin{gathered}
x=\frac{24+2 d_{1}^{2}-d_{2}^{2}-d_{3}^{2}}{8} \\
y=\frac{24-d_{2}^{2}-d_{3}^{2}}{8}
\end{gathered}
$$

Since the received signal power strength is affected by propagation loss and it is sensitive to channel interference, attenuation and reflection, the reader is prone to erroneous readings, because the intersection point is affected by the disturbed RSSI values. The RSSI values circles will not intersect with a single common point (at edges). Figure 4 shows two circles A and B are intersecting at a point, but circle $\mathrm{C}$ is not. Likewise, Figure 5 shows three circles that do not intersect at a single common point. The intersection of all the circles at the edges is the ideal condition which gives accurate estimate. This work estimates the best intersection point between the three circles on conditions that the edge to edge intersection is not available. This is achieved by expanding and reducing trilateration circle sizes assuming that the RSSI pattern distribution is the same/even on all regions. The expansion and reduction are based on a common factor. The aim of expanding and reducing circle sizes is to get the expansion/reduction factor in order to 
understand the difference/magnitude of circles not intersecting.

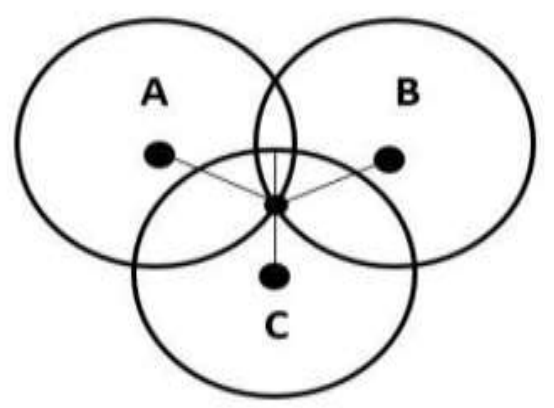

Figure 4: Intersection of two circles A and B.

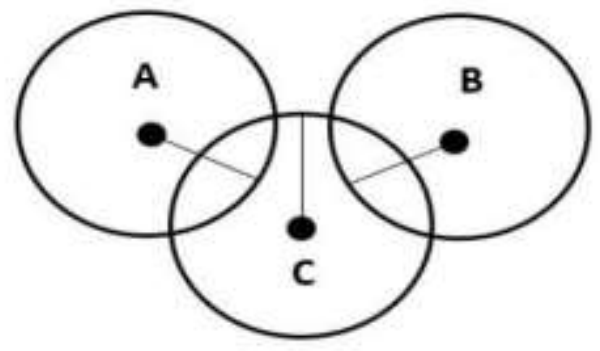

Figure 5: No common intersection point of the circles A, B and C

\section{Position algorithm}

The algorithm starts with the distance obtained from RSSI-distance relationship which is used to check if the three radii intersect. To check if the three circles intersect, normal circle properties are used as shown in Equation 10. If the distance obtained from RSSI measurements are $d_{1}, d_{2}$ and $d_{3}$ for circle $\mathrm{A}, \mathrm{B}$ and $\mathrm{C}$, respectively, then the geometric rules in Figure 6 are used to check if there is intersection. If two circles intersect, then the distance between their center points, should be smaller than the sum of their respective radii.

$$
\begin{aligned}
& r_{1}+r_{2}>d_{1} \\
& r_{3}+r_{2}>d_{2} \\
& r_{1}+r_{3}>d_{3}
\end{aligned}
$$




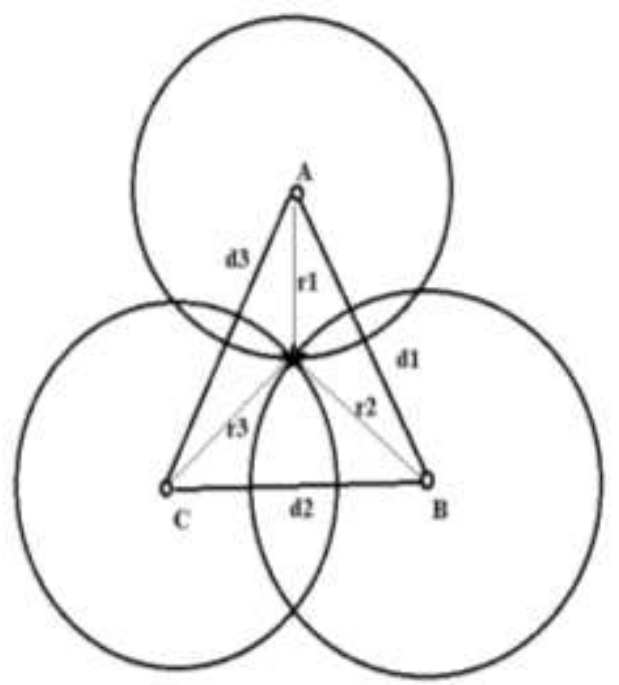

Figure 6: The reduced intersection point of the circles A, B and C.

If there is no intersection at single common point, the three radii are increased by factor " $\mathrm{m}$ ", then the intersection is checked again. The process is repeated until the intersection is found. After the intersection is found, the radii are then reduced by the factor " $\mathrm{k}$ " until there is no intersection. This process assures that the three circles intersect at closest to the edges to give proper position estimation. The value " $m$ " signifies the required condition for the three circles to intersect and is presented as the difference between the distances from reference tag and the computed distances from RSSI as shown in Figure 6. If the circles perfectly intersect, the value " $m$ " will be equal to zero. To get accurate results under different conditions, the value " $\mathrm{m}$ " has to be as close to zero as possible. The value " $\mathrm{m}$ " is always positive and is estimated as the minimum value between the three equations as shown in Equation 11, while the value of " $\mathrm{k}$ " is the minimum value after circle expansion and intersection.

$$
m=\min \left\{\begin{array}{l}
d_{1}-r_{1}-r_{2} \\
d_{2}-r_{2}-r_{3} \\
d_{3}-r_{2}-r_{1}
\end{array}\right\}
$$

Figure 7 shows the developed algorithm which guarantees the best position estimation regardless of the environmental change. 


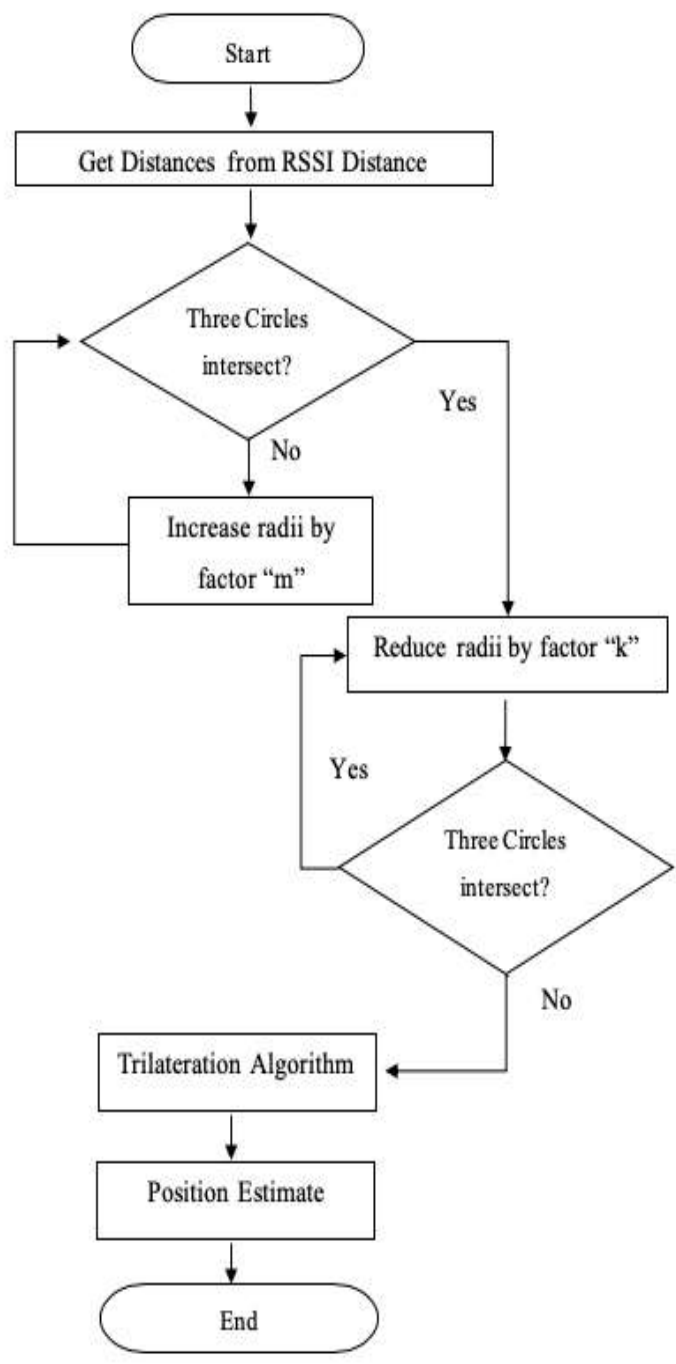

Figure 7: Flowchart of the proposed positioning algorithm.

\section{Environmental modeling}

The theoretical propagation model was developed using CASTALIA platform. The widely used propagation model for RSSI ranging in indoor environments is called lognormal propagation presented in Zou et al. (2013). The model has also been used to model wireless environments in Viswanathan (2020). The model is represented as

$$
\begin{aligned}
& P_{R X}[d B]=P L\left(d_{0}\right)-10 . \eta \cdot \log _{10} \frac{d}{d_{0}}+X_{\sigma}, \\
& d>d_{0}
\end{aligned}
$$

where $\operatorname{PL}\left(d_{0}\right)$ is the path loss value for a reference distance $d_{0}, \eta$ is the path loss coefficient, and $X_{\sigma}$ denotes a fading effect which is a Gaussian random variable with zero 
mean and variance $\sigma$, that models the random variation of the RSSI value. $X_{\sigma}=0$ for LOS transmission.

The wireless channel was established by implementing the average path loss model using NED language in CASTALIA 3.0. The average path loss model emulates the real physical indoor environment with line of sight. Radio module was designed by changing the radio module parameter files on Castalia framework. The module defines the receiver sensitivity, modulation scheme, transmitting power, power transmission matrices, sleep levels. The RFID network was accomplished by using NED file. The network consisted of three RFID active tags and one RFID reader. CASTALIA requires a routing protocol to emulate the network. The bypass routing was chosen for this case. The routing protocol broadcasts the packets to the receiving end which were enough to realize our application. Upon packet receiving, the reader/receiver measures RSSI values from the sender and uses the RSSI information to estimate its current position. The tags were fixed at coordinates $(3,5),(1,3)$ and $(3,1)$. The target tag was allowed to be positioned on different positions in $6 \times 4$ square meter area.

The algorithm was then developed using $\mathrm{C}++$ language. The algorithm takes current radio/network parameters (RSSI and Tags Positions) and uses them in position estimation and error calculation. The algorithm was implemented to fit the environment with path loss coefficient equal to 3.11. The environmental changes were then introduced to the environment using an initialization file. The algorithm that was implemented to fit the environment with path loss coefficient equals to 3.11 and then tested with path loss coefficient equal to 3.11 and 3.21. The results were then passed to Microsoft Excel for further data analysis. To measure robustness, position error, average position error and percentage change on average position error in given environment were calculated and studied. To show robustness, the developed robust algorithm has to show smaller average position error compared to the basic RSSI algorithm with respect to environmental changes.

\section{Results and Discussion}

To evaluate the performance of the proposed algorithm, position and average position error metrics were used. The position and average position error were measured as

$$
\begin{gathered}
P E=\sqrt{\left(x-x_{i}\right)^{2}+\left(y-y_{i}\right)^{2}} \\
A P E=\frac{1}{n} \sum_{i=1}^{n} \sqrt{\left(x-x_{i}\right)^{2}+\left(y-y_{i}\right)^{2}}
\end{gathered}
$$

where, APE is average position error, PE is position error, and $\mathrm{n}$ is number of position estimates. $x_{i}$ and $y_{i}$ are the actual values on the $i^{\text {th }}$ iteration, $\mathrm{x}$ and $y$ are the estimated coordinates of the target node.

Figures 8, 9, 10 and 11 summarize the results showing the position error and the relationship between the algorithm on two environments for the developed robust RSSIbased algorithm for RFID indoor positioning systems and basic RSSI-based algorithm for indoor positioning systems (Javaid et al. 2015). Figure 8 shows position errors of a basic RSSIBased algorithm and the proposed robust RSSIbased algorithm on the respective positions. The average position error of $1.1053 \mathrm{~m}$ was found on proposed algorithm while $1.2448 \mathrm{~m}$ was found on basic RSSI-based algorithm at path loss coefficient (PL) equal to 3.11. Then, environmental changes were introduced by changing the path loss coefficient to 3.21. Figure 9 shows the results obtained after the changes were introduced. The developed robust RSSI-based algorithm showed average position error of $1.1363 \mathrm{~m}$ while basic RSSI-based algorithm for RFID IPS showed the average position error of $1.1803 \mathrm{~m}$. Figures 10 and 11 show the relationship between position errors on given environmental change for basic RSSI based algorithm and proposed robust algorithm, respectively. The plots show how position error of the algorithms changes as the environments change. 


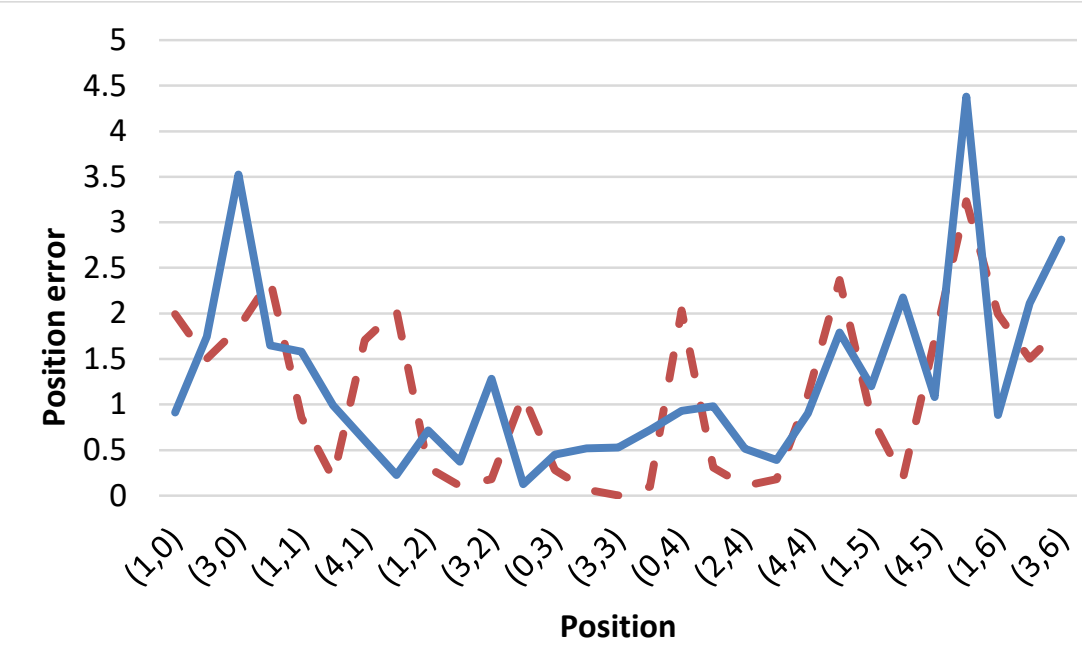

- Proposed Algorithm Basic RSSI-Based Algorithm

Figure 8: Positioning error at path loss equal to 3.11 .

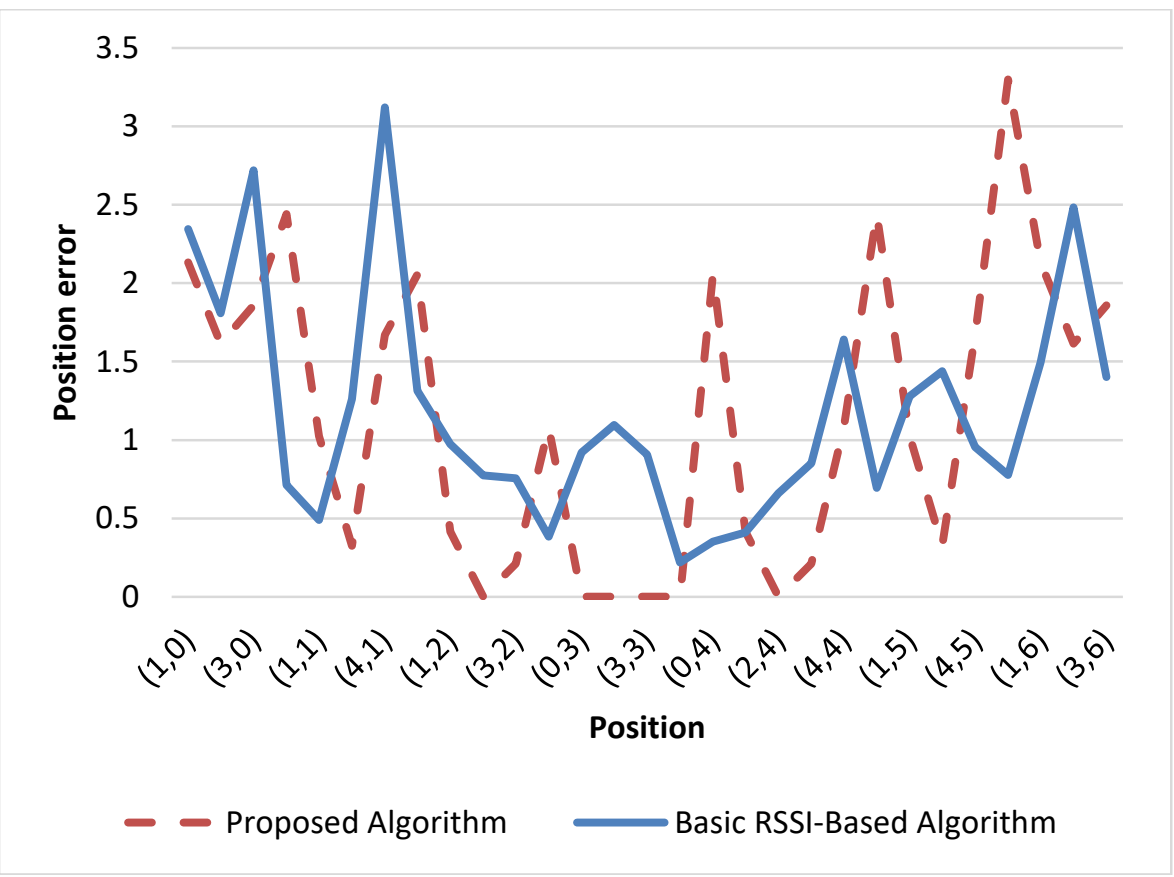

Figure 9: Positioning error at path loss equal to 3.21 . 


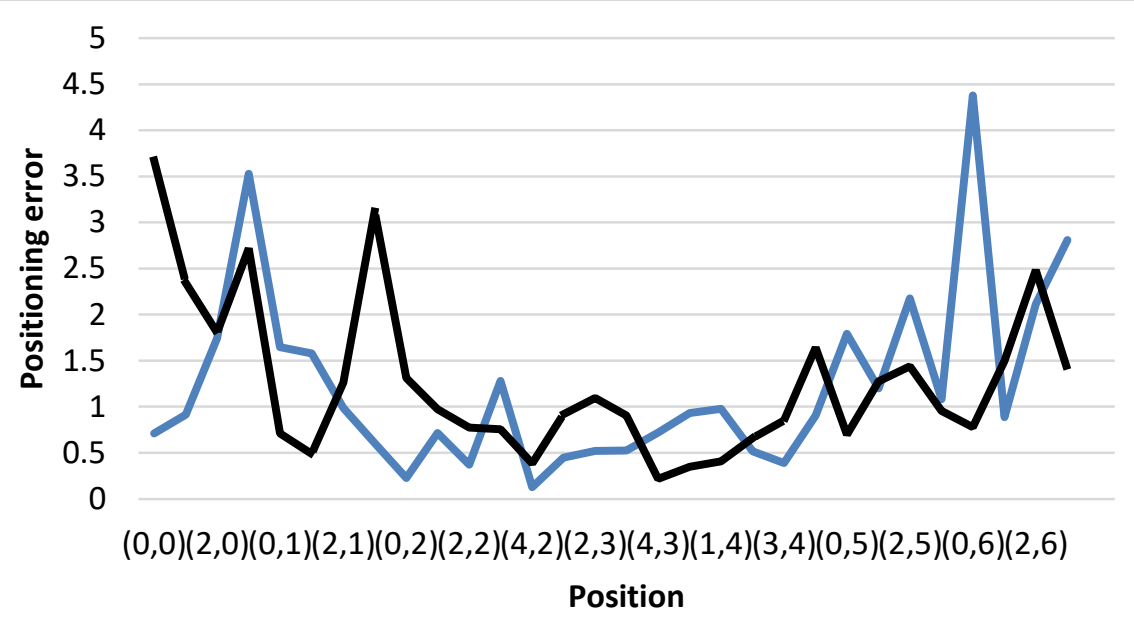

$\longrightarrow \mathrm{PL}=3.11 \longrightarrow \mathrm{PL}=3.21$

Figure 10: Position error relationship on basic RSSI-based algorithm in two different environments $(\mathrm{PL}=3.11$ and $\mathrm{PL}=3.21$.

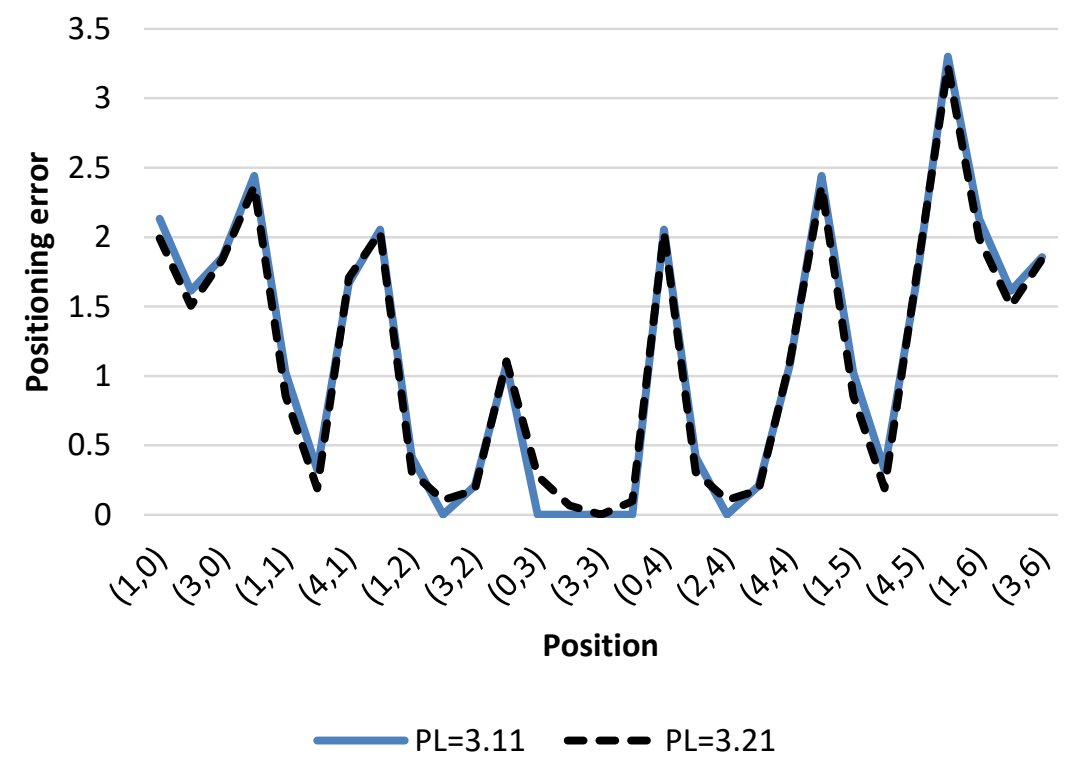

Figure 11: Position error relationship on proposed algorithm in two different environments. 
By having less average error, Figure 8 shows that the robust algorithm is $11.2066 \%$ more accurate than the basic RSSI-based position algorithm under the given specific environment. The changes (nature) in the indoor environment are inevitable. The changes in environment with path loss coefficient of 3.21 show a position error of $1.1363 \mathrm{~m}$ for developed algorithm and $1.1803 \mathrm{~m}$ for the basic algorithm in Figure 9. This shows that the developed robust algorithm has $3.7279 \%$ less position error compared to the basic RSSIbased algorithm for RFID IPS in position estimation on the given environment change. Figure 10 shows that position errors of the basic algorithm fluctuate as the environment changes. However, in Figure 11, the proposed algorithm position error remains almost constant as the environment changes. A robust algorithm has to maintain its error values even under unforeseen conditions. This has been shown in Figure 11, whereby the average increase in error was only $2.7282 \%$ as the environment changes from path loss of 3.11 to 3.21. The average fluctuation in error for basic algorithm is $10 \%$ as observed in Figure 10. The developed robust algorithm uses circles expansion and reduction process. The developed algorithm estimates the target position by estimating the best circle intersection point. The proposed algorithm performs better compared to the basic algorithm by understanding that, there is always a chance that the circles will not intersect at one common point.

\section{Conclusion}

The need of ubiquitous positioning is fueled with current developments on Artificial Intelligence and Internet of Things (IoT) technologies. RFID technology plays a fundamental infrastructure role to fuel these developments. As indoor environment proved to be tedious to model, various researchers have developed algorithms to fit only particular sets of environments. Applications like restaurant self-serving robots, self-moving objects, self-operating drones to tour guiding robots need strong algorithms that will make them robust to environmental changes. This work has introduced a robust RSSI-based algorithm for RFID indoor positioning systems. The algorithm presents remarkable improvements compared to the basic RSSIbased indoor positioning algorithm. The algorithm showed robustness on environmental changes by showing only $2.7282 \%$ change on average positioning error when environmental change was introduced. The new algorithm adapts to different environments without the need of changing values to fit the environmental characteristics.

Testing a robust RSSI algorithm for RFID indoor positioning in real environment is part of future work. This could be achieved by incorporating advanced statistics methods, machine learning approach together with Internet of Things (IoT) to improve the position estimation in the indoor environments. In theory, an ideal indoor positioning algorithm should decrease position error and increase robustness to several environments by making sure that the RSSI values chosen are the strongest and thus giving the best estimation.

\section{References}

Alarifi A, Al-Salman A, Alsaleh M, Alnafessah A, Al-Hadhrami S, Al-Ammar MA and AlKhalifa HS 2016 Ultra wideband indoor positioning technologies: Analysis and recent advances. Sensors 16(5): 707.

Bai YB, Wu S, Wu H and Zhang K 2012 Overview of RFID-based indoor positioning technology. CEUR Workshop Proceedings.

Bekkelien A 2012 Bluetooth indoor positioning. Master thesis, University of Geneva.

Blankenbach J, Norrdine A and Hellmers H 2012 Robust and precise 3D indoor positioning system for harsh environments. IEEE Int. Conf. Indoor Positioning and Indoor Navigation. 1-8.

Carotenuto R, Merenda M, Iero D and Della Corte FG 2018 An indoor ultrasonic system 
for autonomous 3-D positioning. IEEE Trans. Instrum. Meas. 68(7): 2507-2518.

Carrasco U, Coronado PDU, Parto $\mathrm{M}$ and Kurfess T 2018 Indoor location service in support of a smart manufacturing facility. Computers in Industry 103: 132-140.

Chai S, An R and Du Z 2016 An indoor positioning algorithm using bluetooth low energy RSSI. International Conference on Advanced Materials Science and Environmental Engineering, Chiang Mai, Thailand (pp. 26-27).

Chao CT, Chung MH, Chiou JS and Wang CJ 2016 A simple interface for 3D position estimation of a mobile robot with single camera. Sensors 16: 435.

Chen Y, Guo D, Cui W and Li J 2015 Selfadaptive Wi-Fi indoor positioning model. In IEEE International Conference on Geoinformatics, 2-7.

Dao TH, Le MT and Nguyen QC 2014 Indoor localization system using passive UHF RFID Tag and Multi-antennas. IEEE Int. Conference Advanced Technol. Commun. 579-584.

Deng ZA, Wang G, Qin D, Na Z, Cui Y and Chen J 2016 Continuous indoor positioning fusing Wi-Fi, smartphone sensors and landmarks. Sensors 16(9): 1427.

Farahani S 2008 ZigBee Wireless Networks and Transceivers. Elsevier, Newness Imprint of Butterworth-Heinemann Ltd.

Ge B, Wang K, Han J and Zhao B 2015 Improved RSSI positioning algorithm for coal mine underground locomotive. $J$. Elect. Comput. Eng. 2015.

Hausman S and Januszkiewicz Ł 2014 Impact of indoor environment on path loss in body area networks. Sensors 14(10): 1955119560.

Helander F and Hampus E 2015 Evaluation and testing of techniques for indoor positioning. Master thesis, University of Lund.

Huang Y, Zheng J, Xiao, Y and Peng M 2015 Robust localization algorithm based on the RSSI ranging scope. Int. J. Distrib.Sens. Netw. 11(2): 587318.
Hussein AA, Rahman TA and Leow CY 2015 Performance evaluation of localization accuracy for a log-normal shadow fading wireless sensor network under physical barrier attacks. Sensors 15(12): 3054530570 .

Huynh SM, Parry D, Fong ACM and Tang J 2014 Novel RFID and ontology based home localization system for misplaced objects. IEEE Trans. Consum. Electr. 60(3): 402-410.

Javaid R, Qureshi R and Enam RN 2015 RSSI based node localization using trilateration in wireless sensor network. Bahria Univ. J. Inf. Commun. Technol. 8(2): 58-64.

Kaluža M, Beg K and Vukelić B 2017 Analysis of indoor positioning systems. J. Polytech. Rijeka 5(1): 13-32.

Kim HS, Seo W and Baek KR 2017 Indoor positioning system using magnetic field map navigation and an encoder system. Sensors 17(3): 651.

Larranaga J, Muguira L, Lopez-Garde JM and Vazquez JI 2010 An environment adaptive Zigbee-based indoor positioning algorithm. IEEE Int. Conf. Indoor Positioning and Indoor Navigation, 15-17.

Lee SH, Lim IK and Lee JK 2016 Method for improving indoor positioning accuracy using extended Kalman filter. Mobile Inf. Syst. 2016.

Leitinger E, Fröhle M, Meissner P and Witrisal K 2014 Multipath-assisted maximumlikelihood indoor positioning using UWB signals. IEEE Int. Conf. on Comm. 170-175.

Li H 2014 Low-cost 3D Bluetooth indoor positioning with least square. Wireless Pers. Commun. 78. 1331-1344.

Liu X, Shannon J, Voun H, Truijens M, Chi HL and Wang X 2014 Spatial and temporal analysis on the distribution of active radiofrequency identification (RFID) tracking accuracy with the kriging method. Sensors 14(11): 20451-20467.

Liu X, Wen M, Qin G and Liu R 2016 LANDMARC with improved k-nearest algorithm for RFID location system. IEEE 
Int. Conf. Comput. Commun. (ICCC), 25692572.

$\mathrm{Lu}$ YS, Lai CF, Hu CC, Huang YM and Ge XH 2010 Path loss exponent estimation for indoor wireless sensor positioning. KSII $J$. Trans. Internet Info. Syst. 4(3): 243-257.

Maheepala M, Kouzani AZ and Joordens MA 2020 Light-based indoor positioning systems: a review. IEEE Sens. J. 20(8): 3971-3995.

Maung NAM and Zaw W 2020 Comparative study of RSS-based indoor positioning techniques on two different Wi-Fi frequency bands. IEEE Int. Conf. Electrical Engineering/Electronics, Computer, Telecommunications and Information Technology, 185-188.

$\mathrm{Pu} \mathrm{C}, \mathrm{Pu} \mathrm{C}$ and Lee H 2011 Indoor location tracking using received signal strength indicator. In: Emerging Communications for Wireless Sensor Networks, IntechOpen, pp. 229-256.

Sangthong J, Thongkam $\mathrm{J}$ and Promwong $\mathrm{S}$ 2020 Indoor wireless sensor network localization using RSSI based weighting algorithm method. IEEE Int. Conf. Engineering, Applied Sciences and Technology, 1-4.

Tamas J and Toth Z 2019 Topology-based evaluation for symbolic indoor positioning algorithms. IEEE Trans. Industry Appl. 55(6): 6324-6331.

Tsang PYP, Wu CH, Ip WH, Ho GTS and Tse Y K 2015 A bluetooth based indoor positioning system: a simple and rapid approach. Annual Journal IIE (HK). 35(2014): 11-26.

Viswanathan M 2020 Wireless Communication Systems in Matlab. $2^{\text {nd }}$ ed, Independently Published.
Xu H, Ding Y, Wang R, Shen W and Li P 2016 A novel radio frequency identification three-dimensional indoor positioning system based on trilateral positioning algorithm. J. Algor. Comput. Technol 10(3): 158-168.

Xu H, Ding Y, Li P, Wang R and Li Y 2017 An RFID indoor positioning algorithm based on bayesian probability and Knearest neighbor. Sensors 17(8): 1806.

$\mathrm{Xu}$ W, Zhang Z and Wan G 2013 An RSSIbased differential correlation algorithm for wireless node localization. J. Open Automatic Control Syst. 11(5): 73-79.

Yang C and Shao HR 2015 WiFi-based indoor positioning. IEEE Comm. Mag. 53. 150157.

Yayan U, Yucel H and Yazici A 2015 A low cost ultrasonic based positioning system for the indoor navigation of mobile robots. $J$. Intell. Robot. Syst. 78. 541-552.

Zhang H, Zhang J and Wu H 2012 An adaptive localization algorithm based on RSSI in wireless sensor networks. IEEE $2^{\text {nd }}$ Int. Conf. Cloud Computing and Intel. Systems 3: 1133-1136.

Zhang J, Tian GY, Marindra AMJ, Sunny AI and Zhao AB 2017 A review of passive RFID tag antenna-based sensors and systems for structural health monitoring applications. Sensors 17(2): 265.

Zou H, Wang H, Xie L and Jia QS 2013 An RFID indoor positioning system by using weighted path loss and extreme learning machine. IEEE Conf. Cyber-Physical Systems, Networks, and Applications, 6671. 